\title{
Adding a C-terminal Cysteine (CTC) Can Enhance the Bactericidal Activity of Three Different Antimicrobial Peptides
}

\author{
Heng-Li Chen ${ }^{1}$, Pei-Yi Su${ }^{1}$, Shu-Chen Kuo ${ }^{2}$, Tsai-Ling Y. Lauderdale ${ }^{2}$ and Chiaho Shih ${ }^{1 *}$ \\ ${ }^{1}$ Institute of Biomedical Sciences, Academia Sinica, Taipei, Taiwan, ${ }^{2}$ National Institute of Infectious Diseases and \\ Vaccinology, National Health Research Institutes, Zhunan, Taiwan
}

OPEN ACCESS

Edited by:

Octavio Luiz Franco,

Universidade Católica de Brasilia,

Brazil

Reviewed by:

Piyush Baindara,

National Centre for Biological

Sciences, India

Younes Smani,

Instituto de Biomedicina de Sevilla

(IBIS), Spain

*Correspondence:

Chiaho Shih

cshih@ibms.sinica.edu.tw

Specialty section

This article was submitted to

Antimicrobials, Resistance

and Chemotherapy,

a section of the journal

Frontiers in Microbiology

Received: 04 May 2018

Accepted: 11 June 2018

Published: 28 June 2018

Citation:

Chen H-L, SU P-Y, Kuo S-C Lauderdale T-LY and Shih C (2018) Adding a C-terminal Cysteine (CTC) Can Enhance the Bactericidal Activity of Three Different Antimicrobial

Peptides. Front. Microbiol. 9:1440.

doi: 10.3389/fmicb.2018.01440
The emergence of antibiotic-resistant bacteria has threatened our health worldwide. There is an urgent need for novel antibiotics. Previously, we identified a novel $37-$ mer antimicrobial peptide (AMP), HBcARD, with broad spectrum antimicrobial activity. Here, we improved the efficacy of HBcARD, by re-engineering the peptide, including the addition of a new cysteine to its C-terminus (CTC). The new 28-mer derivative, D-150-177C, contains all D-form arginines, in addition to a C-terminal cycteine. This peptide can kill antibiotic-resistant clinical isolates of Gram-negative bacteria, and is more potent than the parental HBcARD peptide in a mouse sepsis model. In another lung infection mouse model, D-150-177C showed protection efficacy against colistinresistant Acinetobacter baumannii. Unlike colistin, we observed no acute toxicity of D-150-177C in vivo. Interestingly, we found that CTC modification could enhance the antibacterial activity of several other AMPs, such as buforinll and lysin. The potential application and mechanism of this CTC method as a general approach to improving drug efficacy, warrants further investigation in the future.

Keywords: antimicrobial peptide, drug resistance, A. baumannii, S. aureus, sepsis, lung infection

\section{INTRODUCTION}

Antibiotics have been used for the treatment of bacterial infection for more than 60 years. Over the past few decades, the extensive use of antibiotics has caused the rapid increase of drug resistance in both Gram-negative and Gram-positive bacteria. There are several life-threatening antibiotics"ESKAPE" pathogens, which caused the majority of nosocomial infection, including Enterococcus faecium, Staphylococcus aureus, Klebsiella pneumoniae, Acinetobacter baumannii, Pseudomonas aeruginosa, and Enterobacter species (Rice, 2008). This situation was headlined as "Bad bugs, No drugs" by The Infectious Diseases Society of America (Boucher et al., 2009). It is therefore urgent to develop new antibiotics for clinical treatment.

Antimicrobial peptides (AMPs) play an important role in the host defense against pathogenic microbes (Zasloff, 2002; Mansour et al., 2014). To date, more than 2,500 natural AMPs have been discovered from various species, such as fungi, plants, and animals (Wang et al., 2016). One common feature of many AMPs, including melittin, defensin and magainin, is to adopt an amphipathic structure on the membrane of microbes. New derivatives with improved potency have largely been based on the structure-activity relationship, by using these natural AMPs as a reference 
template (Zelezetsky and Tossi, 2006; Fjell et al., 2012). Chimeric AMPs, fused from two different AMPs, have also been shown to improve the antimicrobial activity (Fox et al., 2012; Joshi et al., 2012; Ji et al., 2017). Other successful examples of AMP modification include substitutions with D-form amino acids, $\beta$-naphthylalanine, $\alpha, \alpha$-dialkyl amino acids and peptoids (Taira et al., 2010; Yu et al., 2011; Mojsoska et al., 2015; Chen et al., 2016; Khara et al., 2016). In summary, AMPs could be the nextgeneration antibiotics to overcome the problem of antibioticresistance (Hancock and Sahl, 2006; Nguyen et al., 2011; Afacan et al., 2012; Omardien et al., 2016; Ageitos et al., 2017).

We previously identified a novel AMP from human hepatitis $B$ virus core $(\mathrm{HBc})$ protein arginine-rich domain $(\mathrm{ARD})$. This HBcARD peptide exhibited a broad spectrum antimicrobial activity against both Gram-negative and Gram-positive bacteria (Chen et al., 2013). A. baumannii is one of the most common nosocomial infection worldwide (Dijkshoorn et al., 2007; Kuo et al., 2012). Colistin and polymyxin B have been considered as the last hope antibiotics against Gram negative bacteria (Cai et al., 2015). We demonstrated previously that the HBcARD peptide can kill four out of four colistin-resistant A. baumannii in vitro (Chen et al., 2013). A lung infection mouse model by A. baumannii was established previously (Yang et al., 2016). It remains to be tested whether $\mathrm{HBCARD}$ peptide can show any protection efficacy in the mouse model infected with colistinresistant $A$. baumannii.

To further improve the potency of our HBcARD AMP, we designed in this study a series of shorter derivative modified from the parental 37-mer HBcARD. By adding a cysteine at the carboxyl terminus (CTC) of a 27-mer peptide D-150-176, we designed a novel peptide D-150-177C, which can protect mice from death, when infected with either Gram-positive S. aureus or Gram-negative A. baumannii. This novel CTC modification strategy was also able to improve the antibacterial activities of other AMPs, such as buforin and lysin. The potential mechanism of efficacy enhancement by the CTC modification warrants further investigation.

\section{MATERIALS AND METHODS}

\section{Ethics Statement}

This study was carried out in accordance with the recommendations stated in the Guide for the Care and Use of Laboratory Animals, National Research Council, 1996. All animal experiments were conducted under protocols approved by Academia Sinica Institutional Animal Care \& Utilization Committee (ASIACUC permit number 12-02-322) and Institutional Animal Care and Use Committee of National Health Research Institutes (NHRI-IACUC-104139).

\section{Bacterial Isolates}

The antimicrobial activities of HBcARD peptides were tested using a number of bacterial strains, including Pseudomonas aeruginosa Migula strains (ATCC 27853, and ATCC 9027), Klebsiella pneumoniae strain (ATCC 13884), Escherichia coli strain (ATCC 25922), Staphylococcus aureus strains (ATCC
25923, ATCC 29213, and ATCC 19636), and Acinetobacter baumannii strains (ATCC 17978, ATCC19606, TCGH 45530, and TCGH 46709). Colistin-resistant clinical isolates TCGH 45530 and TCGH 46709 were obtained from Tzu-Chi Buddhist General Hospital (TCGH) in Taiwan. Twenty drug-resistant, Gram-negative, clinical isolates, including E. coli, K. pneumoniae, A. baumannii, and P. aeruginosa, were obtained from Taiwan Surveillance of Antimicrobial Resistance program (TSAR), National Health Research Institutes (NHRI), Taiwan.

\section{Antimicrobial Assay}

All peptides were purchased from Yao-Hong Biotechnology Inc. (Taipei, Taiwan). The minimum bactericidal concentration (MBC) was determined as described elsewhere (Chen et al., 2016). Briefly, bacteria were grown in Mueller-Hinton ( $\mathrm{MH})$ broth (Difco) to the mid-logarithmic phase at $37^{\circ} \mathrm{C}$, and were diluted to $10^{6} \mathrm{CFU}$ (colony formation unit)/ml in phosphate buffer $(10 \mathrm{mM}$ sodium phosphate and $50 \mathrm{mM}$ sodium chloride, pH 7.2). Peptides were diluted in the same buffer. Fifty microliters of bacteria were mixed with $50 \mu \mathrm{l}$ of serially diluted peptides, followed by incubation at $37^{\circ} \mathrm{C}$ for $3 \mathrm{~h}$ without shaking. At the end of incubation, bacteria were plated on $\mathrm{MH}$ agar. After incubation overnight at $37^{\circ} \mathrm{C}$, the lowest peptide concentration that displayed no bacterial growth (zero colony) was determined as MBC. All peptides were tested in triplicate. For determining the minimum inhibitory concentration (MIC), bacteria were diluted to $10^{6} \mathrm{CFU} / \mathrm{ml}$ in $\mathrm{MH}$ broth. Peptides were also diluted in $\mathrm{MH}$ broth. The bacteria $(50 \mu \mathrm{l})$ were incubated with serially diluted peptides $(50 \mu \mathrm{l})$ at $37^{\circ} \mathrm{C}$ overnight with shaking. Growth of bacteria was measured by the optical density at $600 \mathrm{~nm}$. The lowest peptide concentration, which showed the same OD600 value as the no peptide control, was defined as MIC. All peptides were tested in duplicate.

\section{In Vivo Animal Studies}

Specific pathogen free (SPF) mice were housed in the individually ventilated cage (IVC) with light controlled in 12-h day-night periods and temperature controlled at $25^{\circ} \mathrm{C}$. Each cage contains 5 mice. They were given standard laboratory food and water ad libitum. The sample size of animal experiments was estimated by the "resource equation" method (Charan and Kantharia, 2013).

We determined the in vivo protection efficacy of peptides in two different mouse infection models. In the mouse sepsis model (Chen et al., 2016), 3-week-old male ICR mice ( 20 g) were purchased from BioLASCO (Taiwan). These mice were inoculated intraperitoneally with $S$. aureus ATCC $19636\left(4 \times 10^{6}\right.$ $\mathrm{CFU} /$ mouse). At $2 \mathrm{~h}$ post-inoculation, the mice were randomly separated into 5 groups and received peptides ( 5 or $10 \mathrm{mg} / \mathrm{kg}$ ) or the PBS control, respectively. Each group contained 5 mice. Mortality was monitored daily for 7 days following the bacterial inoculation. This experiment was repeated twice. In the mouse lung infection model (Yang et al., 2016), 6-8-week-old male C57BL/6JNarl mice ( $\sim 27 \mathrm{~g})$ were purchased from National Laboratory Animal Center, Taiwan. The inoculums $\left(3.5 \times 10^{8}\right.$ $\mathrm{CFU} /$ mouse) were prepared by 1:1 mixing of $\mathrm{LB}$ culture and $10 \%$ mucin (Sigma). All mice were randomly separated into 
3 groups and anesthetized by Isoflurane via inhalation, and inoculated intra-tracheally with colistin-resistant A. baumannii TCGH 46709. Two hours after inoculation, the mice were intraperitoneally injected daily with either colistin $(5 \mathrm{mg} / \mathrm{kg})$ (Fishbain and Peleg, 2010) or HBcARD derivative peptides (5 or $10 \mathrm{mg} / \mathrm{kg}$ ), respectively, for three consecutive days. Each group contained 8 mice. Mortality was monitored every $12 \mathrm{~h}$ following the bacterial inoculation. This experiment was repeated twice.

To measure in vivo the acute toxicity, 3-week-old male ICR mice $(\sim 20 \mathrm{~g})$ were purchased from BioLASCO (Taiwan). The mice were randomly separated into 6 groups, and were intraperitoneally injected with D-150-177C at concentrations of $20,40,60$, and $80 \mathrm{mg} / \mathrm{kg}$. A last-line antibiotic, polymyxin B (20 and $50 \mathrm{mg} / \mathrm{kg}$ ), was used as a control. Each group contained 5 mice. At 1 day post-injection, blood sample was collected from each mouse. The liver function of mice was determined by measuring the ALT level in the blood. The survival rates of injected mice were monitored for 7 days.

\section{Statistical Analysis}

Statistical analysis was performed using the Graphpad software. Survival curves of all groups were plotted by the Kaplan-Meier method and analyzed by the log-rank test.

\section{RESULTS}

\section{The Importance of the Terminal Cysteine}

In our previous results (Chen et al., 2013), we found that the antimicrobial activity against $S$. aureus was diminished, when the HBcARD peptide lost the last 8 amino acids (SQSRESQC). To further improve the potency of our lead compound HBcARD, we tested the antimicrobial activity of several HBcARD derivatives, including peptides modified by truncation and D-arginine substitution for L-arginine (Figure 1). The antimicrobial activities of these peptides were determined by minimal bactericidal concentration (MBC). Like the parental peptide HBcARD 147-175 (Chen et al., 2013), the derivative peptides HBcARD 150-176S and HBcARD 150-177Q showed no detectable bactericidal activity against $S$. aureus (Table 1). To mimic the parental peptide HBcARD 147-183 with a cysteine at the carboxyl terminus, we designed another derivative $\mathrm{HBCARD} 150-177 \mathrm{C}$, by replacing the terminal Q (glutamine) residue of HBcARD 150-177Q with a C (cysteine) residue (Figure 1). The results showed that this Q-to-C substitution effectively rescued the bactericidal activity against three $S$. aureus. In addition, HBcARD 150-177C also retained the same spectrum and potency of antimicrobial activity against a number of Gram-negative bacteria (Table 1). We asked next whether the length of HBcARD 150-177C peptide (28mer) can be further reduced. The potency against $S$. aureus ATCC19636 was no longer detectable up to $57.2 \mathrm{mg} / \mathrm{L}$ for peptides 150-171C (20-mer), 157-177C (21-mer), and 164-177C (14-mer). Altogether, these results here indicated that both arginine and C-terminal cysteine are important for antibacterial activity.

\section{Protection Efficacy of Modified HBcARD in Two Animal Models}

As shown in Figure 2A, we compared the in vivo protection efficacies between HBcARD 150-177Q and 150-177C peptides in the ICR mouse sepsis model. The mice $(\sim 20 \mathrm{~g})$ were i.p. (intraperitoneally) inoculated with S. aureus ATCC19636 $\left(4 \times 10^{6} \mathrm{CFU} / \mathrm{mouse}\right)$, followed by treatments with peptides or the PBS control at $2 \mathrm{~h}$ post-inoculation. We monitored the survival rate for 7 days (Figure 2B). All mice treated with PBS (10/10) died at day 1 post-inoculation. Consistent with its low in vitro $\mathrm{MBC}$ against $S$. aureus, administration of L-150-177Q peptide at the dose of $10 \mathrm{mg} / \mathrm{kg}$ showed a very low protection efficacy (2/10 survival). In contrast, administration of L-150-177C peptide at the same dose protected $70 \%$ of mice $(7 / 10)$ from death $(p<0.05)$. It appears that the C-terminal cysteine is very critical for the in vivo protection as well. Next, we compared the in vivo protection efficacy of HBcARD 150-177C peptides containing either Dform or L-form arginines in this same model. The protection efficacy of peptide L-150-177C was $30 \%(3 / 10)$ at $5 \mathrm{mg} / \mathrm{kg}$, whereas D-150-177C can protect all mice (10/10) from death at the same dose (Figure 2B). The results indicated that D150-177C had a stronger protection effect than L-150-177C $(p<0.01)$.

In addition to the Gram-positive $S$. aureus, we performed another mouse lung infection model to investigate the protection of D-150-177C peptide against the Gram-negative A. baumannii (Figure 2C). C57BL/6JNarl mice $(\sim 27 \mathrm{~g})$ were anesthetized and intra-tracheally inoculated with $A$. baumannii $\left(3.5 \times 10^{8}\right.$ $\mathrm{CFU} / \mathrm{mouse})$. At $2 \mathrm{~h}$ post-inoculation, the mice received i.p. injection of either D-150-177C or colistin, respectively, for three consecutive days ( 1 injection per day). In one experiment with colistin-sensitive A. baumannii ATCC17978 strain, the administration of $\mathrm{D}-150-177 \mathrm{C}$ resulted in a survival rate comparable to that of colistin treatment (data not shown). In the other experiments infected with colistin-resistant A. baumannii TCGH 46709 strain, $12.5 \%$ mice $(2 / 16)$ were poorly rescued by colistin $(5 \mathrm{mg} / \mathrm{kg} /$ day $)$. In contrast, peptide D-150-177C at the dose of 5 and $10 \mathrm{mg} / \mathrm{kg} / \mathrm{day}$ protected $62.5 \%(10 / 16, p<0.01)$ and $87.5 \%(14 / 16$, $p<0.001$ ) of mice from death, respectively (Figure 2D). These results indicated that D-150-177C exhibited a better protection efficacy than colistin for the colistin-resistant A. baumannii.

\section{Peptide D-150-177C Showed Very Low in Vivo Toxicity Than Polymyxin B}

Polymyxins are the last-line antibiotics, but could be toxic at higher dose. We compared the acute toxicity of peptide D-150$177 \mathrm{C}$ with polymyxin $\mathrm{B}$ using ICR mice. The mice ( $20 \mathrm{~g})$ were i.p. injected with D-150-177C peptide $(20-80 \mathrm{mg} / \mathrm{kg}$ body weight). Polymyxin B (50 mg/kg body weight) was used as a control antibiotic. As shown in Figure 3A, all mice treated with peptide D-150-177C at the dose of 20 and $40 \mathrm{mg} / \mathrm{kg}$ survived; while at 60 and $80 \mathrm{mg} / \mathrm{kg}$, the survival rates dropped from 100 to $80 \%(4 / 5)$ and $40 \%(2 / 5)$. The $\mathrm{LD}_{50}$ of $\mathrm{D}-150-177 \mathrm{C}$ peptide was 


\begin{tabular}{|c|c|c|c|}
\hline Experiment & HBCARD & length (mer) & amino acid sequence \\
\hline & $147-183$ & 37 & TVVRRRGRSPRRRTPSPRRRRSQSPRRRRSQSRESQC \\
\hline & & & \\
\hline \multirow{3}{*}{ I } & $150-176 S$ & 27 & RRRGRSPRRRTPSPRRRRSQSPRRRRS \\
\hline & $150-177 Q$ & 28 & RRRGRSPRRRTPSPRRRRSQSPRRRRSQ \\
\hline & $150-177 C$ & 28 & RRRGRSPRRRTPSPRRRRSQSPRRRRSC \\
\hline \multirow{3}{*}{ II } & $150-171 C$ & 20 & RRRGRSPRRRTPSPRRRRSC \\
\hline & $157-177 C$ & 21 & RRRTPSPRRRRSQSPRRRRSC \\
\hline & 164-177C & 14 & RRRRSQSPRRRRSC \\
\hline \multicolumn{4}{|c|}{-----------------------------------------------------------------1} \\
\hline \multirow{3}{*}{ III } & D-150-177C & 28 & rrrGrSPrrrTPSPrrrrSQSPrrrrSC \\
\hline & DL-150-177C & 28 & rRrGRSPrRrTPSPrRrRSQSPrRrRSC \\
\hline & LD-150-177C & 28 & RrRGRSPRrRTPSPRrRrSQSPRrRrSC \\
\hline \multicolumn{4}{|c|}{-----------------------------------------------------------------1} \\
\hline \multirow{4}{*}{ IV } & buforinll & 21 & TRSSRAGLQFPVGRVHRLLRK \\
\hline & buforinIIC & 22 & TRSSRAGLQFPVGRVHRLLRKC \\
\hline & Iysin & 31 & NAKDYKGAAAEFPKWNKAGGRVLAGLVKRRK \\
\hline & IysinC & 32 & NAKDYKGAAAEFPKWNKAGGRVLAGLVKRRKC \\
\hline \multicolumn{4}{|c|}{$\begin{array}{l}\text { FIGURE 1 Amino acid sequences of HBcARD peptides tested for bactericidal activity in this study. HBCARD 147-183 is the prototype peptide. The derivative } \\
\text { peptides were synthesized for optimization of bactericidal activity in this study. Uppercase letters indicate L-form amino acids. Lowercase letters indicate D-form } \\
\text { amino acids. For experiment-I, peptides with different C-terminal amino acid compositions (150-176S, 150-177Q, and 150-177C) were compared for the } \\
\text { antibacterial activity in vitro and in vivo. For experiment-II, N-terminus- and C-terminus-truncated HBcARD peptides (150-171C, 157-177C, and 164-177C) were } \\
\text { generated. These peptides contain different arginine contents. For experiment-III, peptides containing all-D-arginine or partial-D-arginine substitution (D-150-177C } \\
\text { DL-150-177C, and LD-150-177C) were compared for their respective bactericidal activity. For experiment-IV, peptides (buforinll, buforinlIC, Iysin and lysinC) were } \\
\text { compared for the enhancement of in vitro antibacterial activity by the CTC method. }\end{array}$} \\
\hline
\end{tabular}

TABLE 1 | Minimum bactericidal concentration (MBC) of HBCARD peptides against various Gram-negative and Gram-positive bacteria.

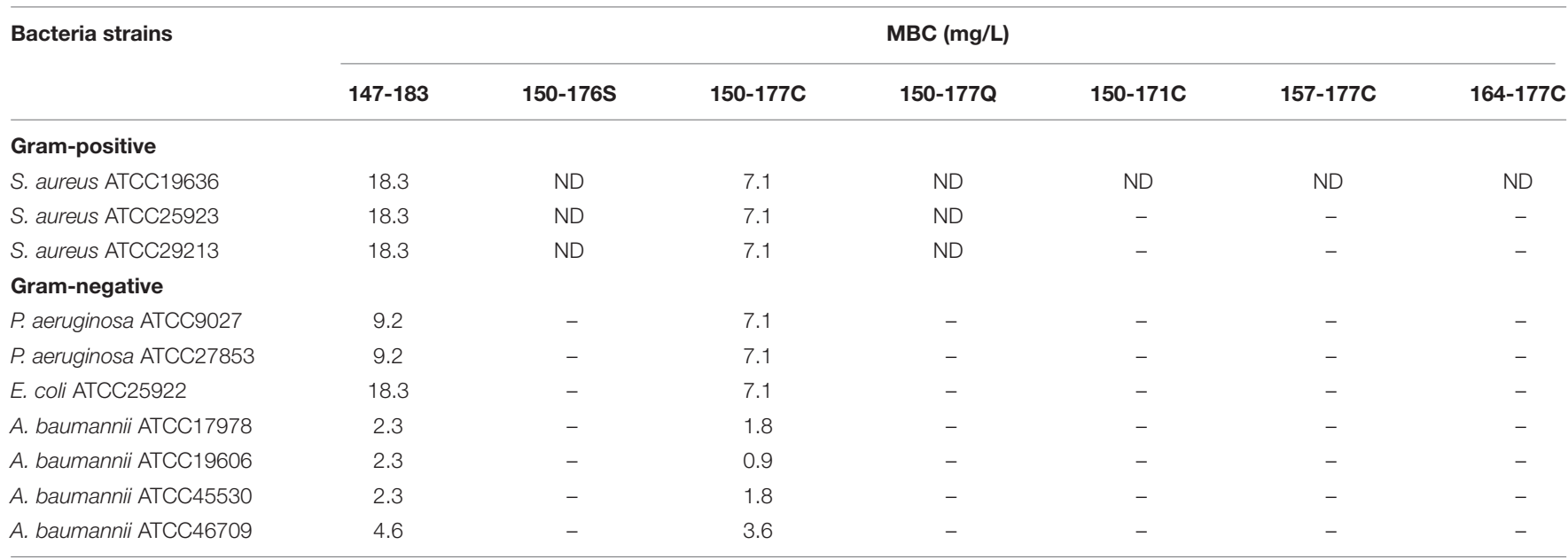

ND, not detectable at the concentration up to $57.2 \mathrm{mg} / \mathrm{L} ;$-, not determined.

estimated to be $75 \mathrm{mg} / \mathrm{kg}$. In contrast, all mice died of polymyxin $\mathrm{B}$ at $50 \mathrm{mg} / \mathrm{kg}$. Therefore, the acute toxicity of peptide D-150$177 \mathrm{C}$ is significantly lower than polymyx B. On the other hand, we also monitored liver injury by measuring serum ALT levels in blood samples from mice at 1 day post-injection. At the dose of $60 \mathrm{mg} / \mathrm{kg}$, D-150-177C peptide showed slightly elevated level of ALT compared to mice treated with $20 \mathrm{mg} / \mathrm{kg}$ polymyxin B (Figure 3B).

\section{Antimicrobial Activity of Peptide D-150-177C Against Clinical Isolates}

We determined the antimicrobial activity of peptide D-150177C against 20 clinical isolates obtained from TSAR (Taiwan Surveillance of Antimicrobial Resistance), including E. coli, K. pneumoniae, A. baumannii, and $P$. aeruginosa. As shown in Table 2, 19 out of 20 isolates were drug-resistant to various antibiotics. Despite their diverse drug-resistant phenotypes, they 
A

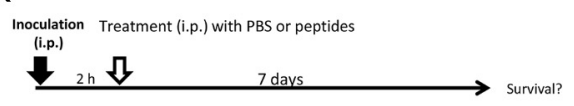

B

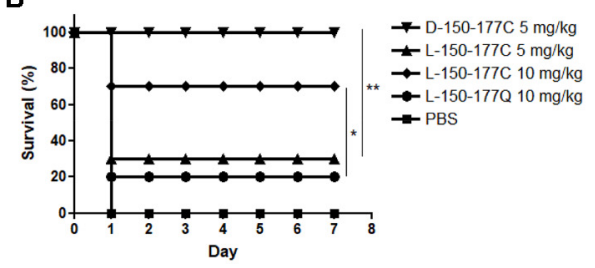

C

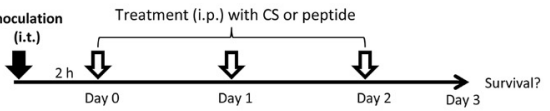

D

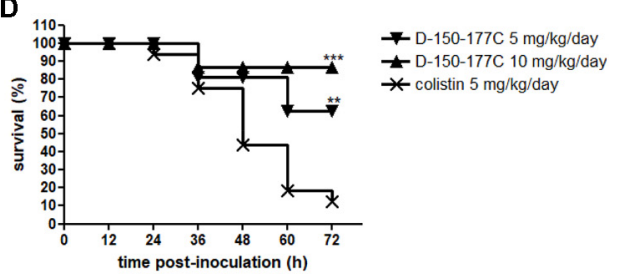

FIGURE 2 | Comparisons of in vivo protection efficacy of HBcARD derivatives in two animal models. (A) In a mouse sepsis model, ICR mice ( $n=10 /$ group) were i.p. inoculated with S. aureus ATCC19636 (4*10\%/mouse) before treatments at $2 \mathrm{~h}$ post-inoculation. The survival rates were monitored for 7 days after injection. (B) All PBS control mice died at day 1, and only 20\% of mice treated with a peptide L-150-177Q survived. Administration of L-150-177C peptide at the dose of 5 and $10 \mathrm{mg} / \mathrm{kg}$ can rescue 30 and $70 \%$ of mice from death, respectively. When treated with $5 \mathrm{mg} / \mathrm{kg}$ of D-150-177C, all mice survived. (C) In a lung infection mouse model, 6-8-week-old male C57BL/6JNarl mice were anesthetized by Isoflurane via inhalation, before intra-tracheal (i.t.) inoculation with $\mathrm{A}$. baumannii (3.5 $\times 10^{8}$ CFU/mouse). Two hours after inoculation, mice were intraperitoneally (i.p.) injected daily with either colistin (CS) or HBcARD peptides for three consecutive days. The survival rates were monitored for 3 days after infection. (D) C57BL/6JNarl mice (16 mice/group) were i.t. inoculated with A. baumannii TCGH46709 (colistin-resistant strain) before treatment with either colistin $(5 \mathrm{mg} / \mathrm{kg}$ ) or D-150-177C peptide (5 and $10 \mathrm{mg} / \mathrm{kg}$ ). There was a dose-dependent effect on the survival rate of mice receiving D-150-177C. The survival rates of mice treated with D-150-177C were significantly higher than that with colistin treatment. This result indicated that D-150-177C was more potent than colistin for killing colistin-resistant $A$. baumannii. ${ }^{*} P<0.05$; ${ }^{* *} P<0.01 ;{ }^{* * *} P<0.001$.

were invariably inhibited by the peptide D150-177C at the range of 16 to $32 \mathrm{mg} / \mathrm{L}$ except for K. pneumoniae.

\section{Comparison of in Vivo Protection Efficacies Among Various Modified 150-177C Peptides}

Peptide D-150-177C contains a total of $14 \mathrm{~L}$-arginines substituted with 14 D-arginines (Figure 1). This modified peptide showed increased antimicrobial activity (Figure 2). Because D-arginine is far more expensive than L-arginine, we investigated the possibility of partial D-arginine substitution in the mouse sepsis model. The in vivo protection efficacies were compared between peptides containing complete or partial D-arginine substitutions. We designed two partial $\mathrm{D}$-arginine-substituted peptides, designated as DL- and LD-150-177C (Figure 1). Both peptide DL-150-177C and all-D-arginine peptide D-150-177C protected all mice from death (Figure 4). While peptide LD-150$177 \mathrm{C}$ appeared to be less protective ( $80 \%$ survival rate), but there were no significant difference between these two $(P>0.05)$.

\section{Enhancement of Antimicrobial Activity via a C-terminal Cysteine (CTC)}

To determine whether the CTC modification strategy can be applied to enhance the spectrum and efficacy of other AMPs, we compared the antibacterial activities between AMPs with and without the CTC modification. We chose buforinII (Park et al., 1998) and lysin (Thandar et al., 2016), whose amino acid sequences bear no resemblance to $\mathrm{HBcARD}$ (Figure 1). As shown in Table 3, buforinIIC exhibited fourfold enhancement over buforinII in the antimicrobial activity against $P$. aeruginosa. This modification also enhanced the antimicrobial activity against both K. pneumoniae and A. baumannii, albeit it showed no effect against $S$. aureus at the concentration up to $1024 \mathrm{mg} / \mathrm{L}$. Similar enhancement of antimicrobial activity by CTC modification was observed in another AMP lysin. Remarkably, the MBC against $P$. aeruginosa, K. pneumoniae, A. baumannii, and $S$. aureus were all significantly enhanced. For example, compared to its parental lysin without a C-terminal cysteine, LysinC exhibited a 4-fold, >8-fold, >8-fold, and 32-fold decrease in MBC against $P$. aeruginosa, K. pneumonia, S. aureus, and A. baumannii, respectively.

\section{DISCUSSION}

Hydrophobic residues in the amphipathic structure of AMPs are known to play an important role in the structure-activity studies (Fjell et al., 2012). However, in this study, we observed similar antimicrobial activity of $\mathrm{HBCARD}$ after deleting three hydrophobic residues (TVV) from the N-terminus (Figure 1 and Table 1). It suggests that these hydrophobic residues at the $\mathrm{N}$-terminus were not required for the antimicrobial activity. At the C-terminus of HBcARD147-183, the last 8 amino acids (SQSRESQC) appeared to be critical for the potency against S. aureus (Chen et al., 2013). It is unclear how these terminal residues could affect the potency. We tested the hypothesis whether the terminal cysteine residue in peptide 150-177C could contribute to the bactericidal activity, by comparing peptides 150-176S, 150-177Q, and 150-177C (Figure 1). Only 150-177C exhibited marked in vitro and in vivo activity against $S$. aureus (Table 1 and Figure 2B). Therefore, the C-terminal cysteine residue (CTC) can enhance the antimicrobial activity of HBcARD peptide against $S$. aureus. In addition to the C-terminal cysteine, 

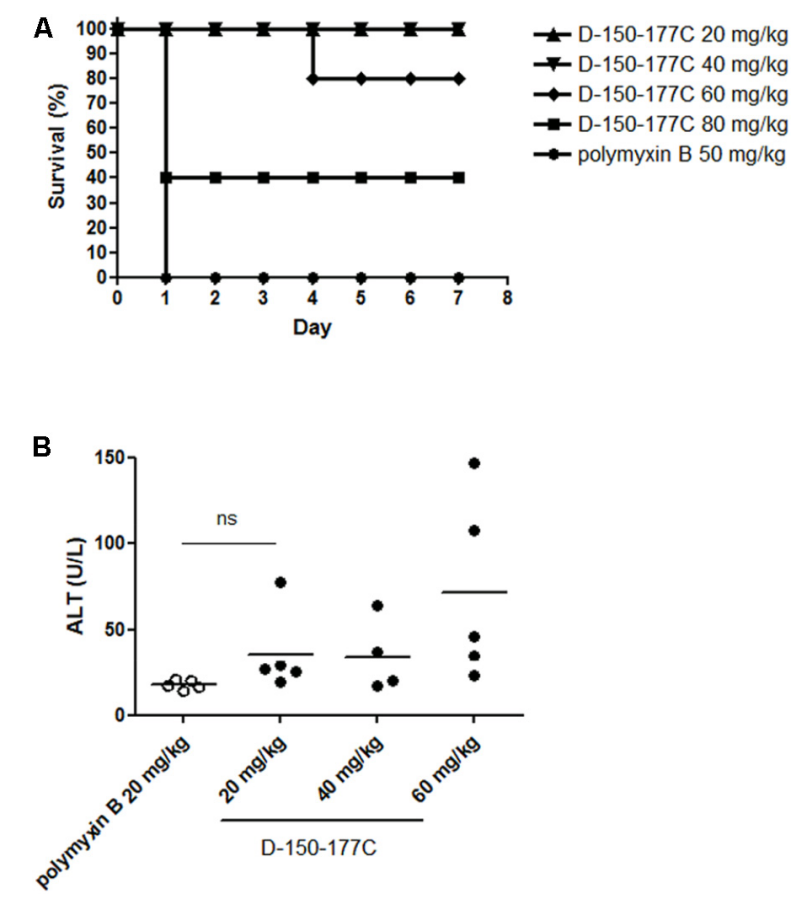

FIGURE 3 | In vivo toxicity of D-150-177C peptide. At day 0, male ICR mice (5 mice/group) were i.p. injected with different doses of D-150-177C peptide (20-80 mg/ $/ \mathrm{kg})$ and polymyxin B $(50 \mathrm{mg} / \mathrm{kg})$, respectively. (A) The survival rates of all groups were monitored for 7 days. All mice treated with polymyxin $B$ (50 mg/kg) died at day 1. In contrast, no acute toxicity was observed in mice treated with 20 and $40 \mathrm{mg} / \mathrm{kg} \mathrm{D}-150-177 \mathrm{C}$. When we increased the dose to 60 and $80 \mathrm{mg} / \mathrm{kg}$, the survival rates of mice were decreased to 80 and $40 \%$. (B) Alanine aminotransferase activity (ALT) was measured in serum samples of mice treated with D-150-177C or polymyxin B at day 1 . The horizontal bars represent the means of ALT values of ICR mice in different experimental groups. Treatment with D-150-177C in the dose of 20 and $40 \mathrm{mg} / \mathrm{kg}$ showed a slight increase in the ALT level than treatment with polymyxin B at $20 \mathrm{mg} / \mathrm{kg}$. The ALT level of mice treated with $60 \mathrm{mg} / \mathrm{kg}$ D-150-177C was significantly higher than that treated with polymyxin B at $20 \mathrm{mg} / \mathrm{kg}(P<0.01)$.

the arginine content appeared to be important as well, based on the deletion mapping experiment (peptides 150-171C, 157177C, and 164-177C). In summary, a C-terminal cysteine and a sufficient number of arginine are important for the HBCARD peptide.

In our previous studies, we successfully improved the in vivo potency of the prototype 37-mer HBcARD by D-arginine substitution in a mouse sepsis model (Chen et al., 2016). Using this same strategy, we demonstrated here that a 28 -mer peptide 150-177C significantly improved the protection efficacy from $30 \%$ by the L-150-177C to $100 \%$ by D-150-177C at the dose of $5 \mathrm{mg} / \mathrm{kg}$ (Figure 2B). So far, our most active derivative is the 28-mer D-150-177C peptide. To reduce the cost for D-amino acids in peptide synthesis, we engineered peptides DL-150-177C and LD-150-177C, which contain only approximately $50 \%$ of $\mathrm{D}$-arginine substitution. These partially $\mathrm{D}$-substituted peptides displayed bactericidal activity similar to peptide D-150-177C, which contains $100 \%$ of $\mathrm{D}$-arginines. It is possible that the protection efficacy can be maintained or improved by further reduction in the number of $\mathrm{D}$-amino acid replacement in the future. Moreover, in our current assay, we measured only the survival rate as the end point. It is worth mentioning here that in our previous studies, bacterial burdens in spleen, liver, and blood were reduced by approximately 100-fold in all tissues in mice treated with full-length HBcARD (Chen et al., 2013).

Drug-resistance bacteria not only can cause therapeutic complications, but also increase the economic burden in hospitals (Perencevich et al., 2003). Therefore, we determined the antimicrobial activity of D-150-177C against 19 clinical isolates with a wide spectrum of drug resistance profiles (Table 2). Except for most strains of K. pneumonia, peptide D-150-177C, in the concentration between 16 and $32 \mathrm{mg} / \mathrm{L}$, inhibited these drugresistant E. coli, A. baumannii, and P. aeruginosa. Because the MIC values between drug-sensitive and drug-resistant strains are similar (Table 2 and data not shown), we observed no crossresistance in vitro from these clinical isolates to D-150-177C.

Polymyxin B and colistin (polymyxin E) are the "lastline" antibiotic peptides currently used in clinical medicine for treatment of drug-resistant Gram-negative bacteria (Cai et al., 2015). However, many cases of colistin- and polymyxin B-resistant strains are emerging, worldwide (Fernandez et al., 2010; Cai et al., 2012; Baadani et al., 2013; Lesho et al., 2013; Lopez-Rojas et al., 2013). A. baumannii is one of the most common antibiotic-resistant pathogens globally, which can cause a wide variety of infections in lung, bloodstream, urinary tract and surgical wounds (Dijkshoorn et al., 2007; Kuo et al., 2012). In our mouse model i.t. infected with colistin-resistant A. baumannii, treatment with D-150-177C can protect $90 \%$ of mice from death (Figure 2D). The lack of cross-resistance in vivo suggested that colistin and D-150-177C must have different modes of action.

A high rate of nephrotoxicity is known to be associated with polymyxins (Doshi et al., 2011; Abdelraouf et al., 2012; Omrani et al., 2015; Roberts et al., 2015). In our acute toxicity mouse model, $80 \%$ of mice receiving $60 \mathrm{mg} / \mathrm{kg}$ peptide $\mathrm{D}-150-177 \mathrm{C}$ survived, while $100 \%$ of mice died of polymyxin B at $50 \mathrm{mg} / \mathrm{kg}$ (Figure 3A). Although the mice receiving peptide D-150-177C $(60 \mathrm{mg} / \mathrm{kg})$ had an elevated ALT level compared to polymyxin B (20 mg/kg) treated mice (Figure 3B), the average ALT value appeared to fall in the range of the basal level (less than $100 \mathrm{IU} / \mathrm{L}$ ) of untreated mice (see Materials and Methods). Taken together, treatment with D-150-177C peptide appeared to be well tolerated in the mouse model. Finally, in our current study, we performed no accumulative toxicity assays. This is because in our current short-term study, we tested only one-shot treatment for the sepsis model, and only three shots in 3 days for the lung infection model (Figure 2).

Ionic strength in the medium has been shown to influence the antibacterial activities of human beta-defensin 3 (hBD-3) derivatives (Boniotto et al., 2003; Kluver et al., 2005). In addition, it has been reported that polyanionic peptides present in LB and BHI media may form electrostatic complexes with cationic polymers, which would decrease the potency by diminishing the binding to the anionic lipopolysaccharide layer of E. coli. (Choi et al., 2014). In our studies, we noted that the MIC values were not always in parallel with the MBC values. For example, the MICs 
TABLE 2 | Minimum inhibitory concentration (MIC) of D-150-177C against antibiotics-resistant bacterial isolates.

\begin{tabular}{|c|c|c|c|c|}
\hline Clinical isolates & & Origin & MIC (mg/L) & Resistance phenotype \\
\hline & 2 & Urine & 16 & AMP, CFZ-UTI, CIP, FEP, FRX, FTX, GEN, LEV \\
\hline & 4 & Urine & 16 & AMK, AMP, ATM, CAZ, CFZ, CIP, FEP, FOX, FRX, FTX, GEN, LEV, SXT \\
\hline & 5 & Urine & 16 & AMP, CAZ, CFZ, COL, FOX, FRX, FTX, SXT \\
\hline \multirow[t]{4}{*}{ K. pneumoniae } & 1 & Liver abscess & $>32$ & AMP \\
\hline & 3 & liver & 32 & AMP \\
\hline & 4 & Urine & $>32$ & AMP, ATM, CAZ, CFZ, CIP, FEP, FOX, FRX, FTX, LEV, TZP \\
\hline & 5 & Liver abscess & $>32$ & AMP \\
\hline \multirow[t]{3}{*}{ A. baumannii } & 1 & Urine & 32 & AMK, CAZ, CIP, DOR, FTX, GEN, IMP, LEV, MEM, PIP, TZP \\
\hline & 2 & Urine & 32 & AMK, CAZ, CIP, DOR, FEP, FTX, GEN, IMP, LEV, MEM, PIP \\
\hline & 3 & Urine & 32 & AMK, AMS, CAZ, CIP, DOR, FEP, FTX, GEN, IMP, MEM, PIP, TZP \\
\hline & 2 & Urine & 32 & ATM, CIP, DOR, IMP, LEV, MEM \\
\hline & 3 & Urine & 16 & ATM, CIP, IMP, LEV, MEM \\
\hline & 4 & Urine & 32 & AMK, CIP, DOR, FEP, GEN, IMP, LEV, MEM, PIP, TZP \\
\hline & 5 & Urine & 32 & ATM, CAZ, IMP, MEM, PIP, TZP \\
\hline
\end{tabular}

AMK, Amikacin; AMP, Ampicillin; AMS, Ampicillin/Sulbactam; ATM, Aztreonam; CAZ, Ceftazidime; CFZ, Cefazolin (based on interpretive criteria for uncomplicated urinary tract infection); CIP, Ciprofloxacin; COL, Colistin; DOR, Doripenem; EPT, Ertapenem; FEP, Cefepime; FOX, Cefoxitin; FRX, Cefuroxime; FTX, Cefotaxime; GEN, Gentamicin; IMP, Imipenem; MEM, Meropenem; PIP, Piperacillin; SXT, Trimethoprim/sulfamethoxazole; TZP, Piperacillin/Tazobactam.

TABLE 3 | Enhancement of antimicrobial activity of buforin and lysin.

\begin{tabular}{lcccc}
\hline Bacteria & \multicolumn{4}{c}{ MBC (mg/L) } \\
\cline { 2 - 5 } & Buforinll & BuforinlIC & Lysin & LysinC \\
\hline P. aeruginosa ATCC9027 & 16 & 4 & 64 & 16 \\
K. pneumoniae ATCC13884 & $\mathrm{ND}$ & $>1024$ & $\mathrm{ND}$ & 128 \\
A. baumannii ATCC17978 & $>1024$ & 1024 & $>1024$ & 32 \\
S. aureus ATCC 19636 & $\mathrm{ND}$ & $\mathrm{ND}$ & $\mathrm{ND}$ & 128 \\
\hline
\end{tabular}

ND, not detectable at the concentration up to $1024 \mathrm{mg} / \mathrm{L}$.

of D-150-177C for A. baumannii were 16-32 mg/L (Table 2), whereas its MBCs for $A$. baumannii were $1-4 \mathrm{mg} / \mathrm{L}$ (Table 1). Comparing to the D-150-177C peptide, colistin has a lower MIC around $4 \mathrm{mg} / \mathrm{L}$ for A. baumannii ATCC17978 strain. Despite their significant difference in the MIC values, we observed similar protection efficacies between colistin and D-150-177C in the animal model i.t. infected with $A$. baumannii. Therefore, the MIC values in vitro do not always reflect the in vivo antibacterial activity. Using the $0.5 \mathrm{x} \mathrm{MH}$ broth, we found that the MIC values became closer to the MBC values (data not shown). Altogether, it is important to include an in vivo assay in an animal model, when the antibacterial activity is being evaluated, as the in vitro MIC assay could be subject to the influence of ionic strength in the broth.

Previously, we demonstrated that the C-terminal octamer peptide SQSRESQC of HBCARD is important for the antibacterial activity against $S$. aureus (Chen et al., 2013). This observation was confirmed recently. When phage lysin was conjugated with

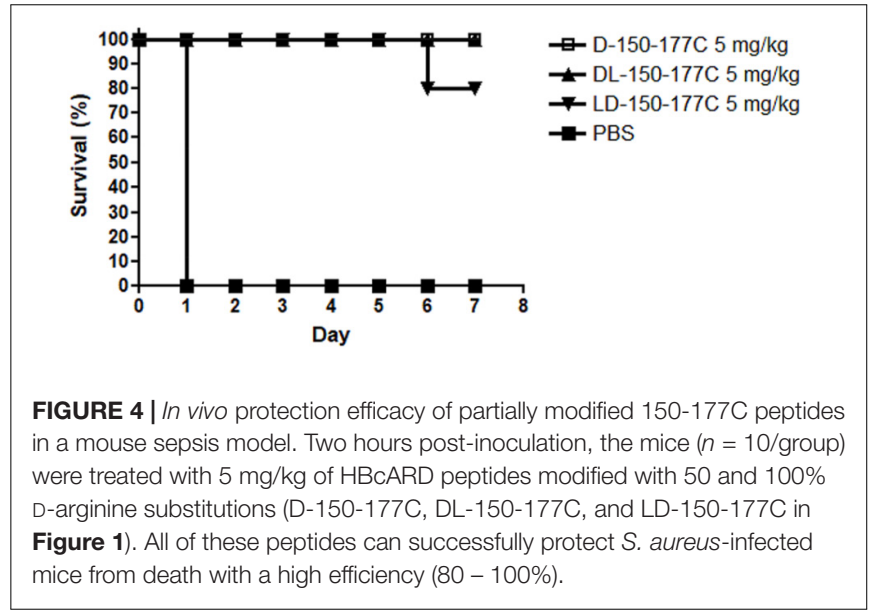

this octamer peptide of HBcARD or a scrambled peptide, the antibacterial activity of phage lysin was enhanced (Thandar et al., 2016). The principal determinant for this activity enhancement has remained ill-defined. In our investigation on the modification of HBcARD, we invented a CTC modification strategy (Table 3). In this approach, antibacterial activity or spectrum, including Gram-positive and Gram-negative, can be improved by adding an exogenous cysteine to the end of the C-terminus of an AMP, regardless of its sequence context. We cited here two such successful examples using the less potent buforinII and lysin. On the other hand, we experienced unsuccessful examples via CTC modifications, such as indolicidin, magainine and epinecidin-1 (data not shown). 
It remains unclear what could be the mechanism for the CTC-mediated enhancement. It is unlikely that the effect is simply due to the peptide dimerization via the cysteine disulfide bond formation. The CTC enhancement effects are variable, depending on the bacteria being treated; however, the increased antibacterial activities are in general far greater than twofold. To date, a rich AMP database includes near three thousand naturally occurring AMPs (Wang et al., 2016). It should be rewarding to explore systematically, whether this simple CTC modification approach could help broaden the spectrum and boost the activity of these AMPs against various drug-resistant microorganisms in clinical medicine.

\section{REFERENCES}

Abdelraouf, K., Braggs, K. H., Yin, T., Truong, L. D., Hu, M., and Tam, V. H. (2012). Characterization of polymyxin B-induced nephrotoxicity: implications for dosing regimen design. Antimicrob. Agents Chemother. 56, 4625-4629. doi: 10.1128/AAC.00280-12

Afacan, N. J., Yeung, A. T., Pena, O. M., and Hancock, R. E. (2012). Therapeutic potential of host defense peptides in antibiotic-resistant infections. Curr. Pharm. Des. 18, 807-819. doi: 10.2174/138161212799277617

Ageitos, J. M., Sanchez-Perez, A., Calo-Mata, P., and Villa, T. G. (2017). Antimicrobial peptides (AMPs): ancient compounds that represent novel weapons in the fight against bacteria. Biochem. Pharmacol. 133, 117-138. doi: $10.1016 /$ j.bcp.2016.09.018

Baadani, A. M., Thawadi, S. I., El-Khizzi, N. A., and Omrani, A. S. (2013). Prevalence of colistin and tigecycline resistance in Acinetobacter baumannii clinical isolates from 2 hospitals in Riyadh Region over a 2-year period. Saudi Med. J. 34, 248-253.

Boniotto, M., Antcheva, N., Zelezetsky, I., Tossi, A., Palumbo, V., Verga Falzacappa, M. V., et al. (2003). A study of host defence peptide beta-defensin 3 in primates. Biochem. J. 374, 707-714. doi: 10.1042/bj20030528

Boucher, H. W., Talbot, G. H., Bradley, J. S., Edwards, J. E., Gilbert, D., Rice, L. B., et al. (2009). Bad bugs, no drugs: no ESKAPE! An update from the Infectious Diseases Society of America. Clin. Infect. Dis. 48, 1-12. doi: 10.1086/595011

Cai, Y., Chai, D., Wang, R., Liang, B., and Bai, N. (2012). Colistin resistance of Acinetobacter baumannii: clinical reports, mechanisms and antimicrobial strategies. J. Antimicrob. Chemother. 67, 1607-1615. doi: 10.1093/jac/dks084

Cai, Y., Lee, W., and Kwa, A. L. (2015). Polymyxin B versus colistin: an update. Expert Rev. Anti Infect. Ther. 13, 1481-1497. doi: 10.1586/14787210.2015. 1093933

Charan, J., and Kantharia, N. D. (2013). How to calculate sample size in animal studies? J. Pharmacol. Pharmacother. 4, 303-306. doi: 10.4103/0976-500X. 119726

Chen, H. L., Su, P. Y., Chang, Y. S., Wu, S. Y., Liao, Y. D., Yu, H. M., et al. (2013). Identification of a novel antimicrobial peptide from human hepatitis $\mathrm{B}$ virus core protein arginine-rich domain (ARD). PLoS Pathog. 9:e1003425. doi: 10.1371/journal.ppat.1003425

Chen, H. L., Su, P. Y., and Shih, C. (2016). Improvement of in vivo antimicrobial activity of HBcARD peptides by D-arginine replacement. Appl. Microbiol. Biotechnol. 100, 9125-9132. doi: 10.1007/s00253-016-7621-6

Choi, H., Chakraborty, S., Liu, R., Gellman, S. H., and Weisshaar, J. C. (2014). Medium effects on minimum inhibitory concentrations of nylon-3 polymers against E. coli. PLoS One 9:e104500. doi: 10.1371/journal.pone.010 4500

Dijkshoorn, L., Nemec, A., and Seifert, H. (2007). An increasing threat in hospitals: multidrug-resistant Acinetobacter baumannii. Nat. Rev. Microbiol. 5, 939-951. doi: $10.1038 /$ nrmicro1789

Doshi, N. M., Mount, K. L., and Murphy, C. V. (2011). Nephrotoxicity associated with intravenous colistin in critically ill patients. Pharmacotherapy 31, 1257-1264. doi: 10.1592/phco.31.12.1257

\section{AUTHOR CONTRIBUTIONS}

H-LC, S-CK, and CS designed the experiments. H-LC, S-CK, $\mathrm{P}-\mathrm{YS}$ performed the experiments. All authors analyzed the data. CS, T-LL, and H-LC prepared the manuscript.

\section{FUNDING}

This research was supported by the grants from Academia Sinica and the Ministry of Science and Technology [MOST 104-021001-09-02, MOST 105-0210-01-13-01, MOST 106-2320-B-001010, MOST 106-0210-01-15-02, and MOST 107-0210-01-19-01], Taiwan.

Fernandez, L., Gooderham, W. J., Bains, M., Mcphee, J. B., Wiegand, I., and Hancock, R. E. (2010). Adaptive resistance to the "last hope" antibiotics polymyxin B and colistin in Pseudomonas aeruginosa is mediated by the novel two-component regulatory system ParR-ParS. Antimicrob. Agents Chemother. 54, 3372-3382. doi: 10.1128/AAC.00242-10

Fishbain, J., and Peleg, A. Y. (2010). Treatment of Acinetobacter infections. Clin. Infect. Dis. 51, 79-84. doi: 10.1086/653120

Fjell, C. D., Hiss, J. A., Hancock, R. E., and Schneider, G. (2012). Designing antimicrobial peptides: form follows function. Nat. Rev. Drug Discov. 11, 37-51. doi: 10.1038/nrd3591

Fox, M. A., Thwaite, J. E., Ulaeto, D. O., Atkins, T. P., and Atkins, H. S. (2012). Design and characterization of novel hybrid antimicrobial peptides based on cecropin A, LL-37 and magainin II. Peptides 33, 197-205. doi: 10.1016/j. peptides.2012.01.013

Hancock, R. E., and Sahl, H. G. (2006). Antimicrobial and host-defense peptides as new anti-infective therapeutic strategies. Nat. Biotechnol. 24, 1551-1557. doi: $10.1038 /$ nbt1267

Ji, S., Li, W., Baloch, A. R., Wang, M., Li, H., Cao, B., et al. (2017). Efficient biosynthesis of a Cecropin A-melittin mutant in Bacillus subtilis WB700. Sci. Rep. 7:40587. doi: 10.1038/srep40587

Joshi, S., Bisht, G. S., Rawat, D. S., Maiti, S., and Pasha, S. (2012). Comparative mode of action of novel hybrid peptide CS-1a and its rearranged amphipathic analogue CS-2a. FEBS J. 279, 3776-3790. doi: 10.1111/j.1742-4658.2012.08738.x

Khara, J. S., Priestman, M., Uhia, I., Hamilton, M. S., Krishnan, N., Wang, Y., et al. (2016). Unnatural amino acid analogues of membrane-active helical peptides with anti-mycobacterial activity and improved stability. J. Antimicrob. Chemother. 71, 2181-2191. doi: 10.1093/jac/dkw107

Kluver, E., Schulz-Maronde, S., Scheid, S., Meyer, B., Forssmann, W. G., and Adermann, K. (2005). Structure-activity relation of human beta-defensin 3: influence of disulfide bonds and cysteine substitution on antimicrobial activity and cytotoxicity. Biochemistry 44, 9804-9816. doi: 10.1021/bi050272k

Kuo, S. C., Chang, S. C., Wang, H. Y., Lai, J. F., Chen, P. C., Shiau, Y. R., et al. (2012). Emergence of extensively drug-resistant Acinetobacter baumannii complex over 10 years: nationwide data from the Taiwan Surveillance of Antimicrobial Resistance (TSAR) program. BMC Infect. Dis. 12:200. doi: 10.1186/1471-233412-200

Lesho, E., Yoon, E. J., Mcgann, P., Snesrud, E., Kwak, Y., Milillo, M., et al. (2013). Emergence of colistin-resistance in extremely drug-resistant Acinetobacter baumannii containing a novel pmrCAB operon during colistin therapy of wound infections. J. Infect. Dis. 208, 1142-1151. doi: 10.1093/infdis/ jit293

Lopez-Rojas, R., Mcconnell, M. J., Jimenez-Mejias, M. E., Dominguez-Herrera, J., Fernandez-Cuenca, F., and Pachon, J. (2013). Colistin resistance in a clinical Acinetobacter baumannii strain appearing after colistin treatment: effect on virulence and bacterial fitness. Antimicrob. Agents Chemother. 57, 4587-4589. doi: 10.1128/AAC.00543-13

Mansour, S. C., Pena, O. M., and Hancock, R. E. (2014). Host defense peptides: front-line immunomodulators. Trends Immunol. 35, 443-450. doi: 10.1016/j.it. 2014.07.004 
Mojsoska, B., Zuckermann, R. N., and Jenssen, H. (2015). Structure-activity relationship study of novel peptoids that mimic the structure of antimicrobial peptides. Antimicrob. Agents Chemother. 59, 4112-4120. doi: 10.1128/AAC. 00237- 15

Nguyen, L. T., Haney, E. F., and Vogel, H. J. (2011). The expanding scope of antimicrobial peptide structures and their modes of action. Trends Biotechnol. 29, 464-472. doi: 10.1016/j.tibtech.2011.05.001

Omardien, S., Brul, S., and Zaat, S. A. (2016). Antimicrobial activity of cationic antimicrobial peptides against gram-positives: current progress made in understanding the mode of action and the response of bacteria. Front. Cell Dev. Biol. 4:111. doi: 10.3389/fcell.2016.00111

Omrani, A. S., Alfahad, W. A., Shoukri, M. M., Baadani, A. M., Aldalbahi, S., Almitwazi, A. A., et al. (2015). High dose intravenous colistin methanesulfonate therapy is associated with high rates of nephrotoxicity; a prospective cohort study from Saudi Arabia. Ann. Clin. Microbiol. Antimicrob. 14:3. doi: 10.1186/ s12941-015-0062-8

Park, C. B., Kim, H. S., and Kim, S. C. (1998). Mechanism of action of the antimicrobial peptide buforin II: buforin II kills microorganisms by penetrating the cell membrane and inhibiting cellular functions. Biochem. Biophys. Res. Commun. 244, 253-257. doi: 10.1006/bbrc.1998.8159

Perencevich, E. N., Sands, K. E., Cosgrove, S. E., Guadagnoli, E., Meara, E., and Platt, R. (2003). Health and economic impact of surgical site infections diagnosed after hospital discharge. Emerg. Infect. Dis. 9, 196-203. doi: 10.3201/ eid0902.020232

Rice, L. B. (2008). Federal funding for the study of antimicrobial resistance in nosocomial pathogens: no ESKAPE. J. Infect. Dis. 197, 1079-1081. doi: 10.1086/ 533452

Roberts, K. D., Azad, M. A., Wang, J., Horne, A. S., Thompson, P. E., Nation, R. L., et al. (2015). Antimicrobial activity and toxicity of the major lipopeptide components of polymyxin b and colistin: last-line antibiotics against multidrugresistant gram-negative bacteria. ACS Infect. Dis. 1, 568-575. doi: 10.1021/ acsinfecdis.5b00085

Taira, J., Kida, Y., Yamaguchi, H., Kuwano, K., Higashimoto, Y., and Kodama, H. (2010). Modifications on amphiphilicity and cationicity of unnatural amino acid containing peptides for the improvement of antimicrobial activity against pathogenic bacteria. J. Pept. Sci. 16, 607-612. doi: 10.1002/psc.1270

Thandar, M., Lood, R., Winer, B. Y., Deutsch, D. R., Euler, C. W., and Fischetti, V. A. (2016). Novel engineered peptides of a phage lysin as effective antimicrobials against multidrug-resistant Acinetobacter baumannii. Antimicrob. Agents Chemother. 60, 2671-2679. doi: 10.1128/AAC.02 972-15

Wang, G., Li, X., and Wang, Z. (2016). APD3: the antimicrobial peptide database as a tool for research and education. Nucleic Acids Res. 44, D1087-D1093. doi: 10.1093/nar/gkv1278

Yang, Y. S., Lee, Y., Tseng, K. C., Huang, W. C., Chuang, M. F., Kuo, S. C., et al. (2016). In vivo and In vitro efficacy of minocycline-based combination therapy for minocycline-resistant Acinetobacter baumannii. Antimicrob. Agents Chemother. 60, 4047-4054. doi: 10.1128/AAC.02994-15

Yu, H. Y., Tu, C. H., Yip, B. S., Chen, H. L., Cheng, H. T., Huang, K. C., et al. (2011). Easy strategy to increase salt resistance of antimicrobial peptides. Antimicrob. Agents Chemother. 55, 4918-4921. doi: 10.1128/AAC.00202-11

Zasloff, M. (2002). Antimicrobial peptides of multicellular organisms. Nature 415, 389-395. doi: 10.1038/415389a

Zelezetsky, I., and Tossi, A. (2006). Alpha-helical antimicrobial peptides-using a sequence template to guide structure-activity relationship studies. Biochim. Biophys. Acta 1758, 1436-1449. doi: 10.1016/j.bbamem.2006.03.021

Conflict of Interest Statement: The authors declare that the research was conducted in the absence of any commercial or financial relationships that could be construed as a potential conflict of interest.

Copyright (c) 2018 Chen, Su, Kuo, Lauderdale and Shih. This is an open-access article distributed under the terms of the Creative Commons Attribution License (CC BY). The use, distribution or reproduction in other forums is permitted, provided the original author(s) and the copyright owner are credited and that the original publication in this journal is cited, in accordance with accepted academic practice. No use, distribution or reproduction is permitted which does not comply with these terms. 